\title{
Forma Indeterminada da Moléstia de Chagas. Proposta de Novos Critérios de Caracterização e Perspectivas de Tratamento Precoce da Cardiomiopatia
}

\author{
José Antonio Marin-Neto, Oswaldo César de Almeida Filho, Antonio Pazin-Filho, \\ Benedito Carlos Maciel \\ Ribeirão Preto, SP
}

\begin{abstract}
O conceito de forma indeterminada tem raízes estabelecidas, muito precocemente, na história do conhecimento da doença de Chagas ${ }^{1}$, abrangendo chagásicos, aparentemente normais, porém com potencial evolutivo. Mas é importante distinguir-se as duas vertentes essenciais desta noção médica. A primeira e de mais simples compreensão, referese ao estado de indivíduos, comprovadamente infectados pelo agente etiológico, nos quais não são detectadas anormalidades clínicas imputáveis à doença de Chagas ${ }^{2}$. A segunda vertente, mais tardiamente incorporada ao conhecimento da moléstia, é mais complexa, e concerne ao período de sua história natural, em que muitos pacientes podem permanecer temporária, ou indefinidamente (durante sua vida) ${ }^{3}$.

Se o conceito sempre existiu - traduzindo essencialmente a ausência de manifestações clínicas da doença após infecção por T. cruzi -, a denominação correspondente tem variado ao longo das várias décadas de estudos e investigações da doença (e.g. forma subclínica, pré-clínica, latente, cardíaca potencial, laboratorial) ${ }^{4}$. Associou-se, precocemente, ao conceito a intrigante incerteza prognóstica pairando sobre os pacientes, que assim se apresentam ${ }^{5}$.

A partir da conotação essencial de ausência de expressão clínica de envolvimento orgânico, registrou-se bastante variação na exata definição de como e quais manifestações focalizar e excluir para caracterizar a forma indeterminada ${ }^{6}$. A despeito dessa incerteza, hoje aceita-se, como consenso tácito, a normatização de considerar a forma indeterminada da doença de Chagas, para efeito de aplicação médica (inclusive com reflexos sociais) e de pesquisa como $^{7}$ : indivíduos chagásicos assintomáticos, nos quais
\end{abstract}

Hospital das Clínicas da Faculdade de Medicina de Ribeirão Preto - USP Correspondência: José Antonio Marin-Neto - Divisão de Cardiologia, Depto. de Clínica Médica - Hospital das Clínicas, FMRP-USP - Av. Bandeirantes, 3900 14048-900 - Ribeirão Preto, SP - E-mail: marin_neto@yahoo.com

Recebido para publicação em $26 / 2 / 02$

Aceito em 27/5/02 se requer a explícita demonstração de normalidade eletrocardiográfica, radiológica cardíaca, e a exclusão de envolvimento esofágico e intestinal colônico por técnicas radiológicas (neste último órgão por meio de exame com enema opaco). Se rigorosamente seguido esse preceito, em investigações dirigidas para explorar formas isoladas (cardíaca e digestiva) da doença de Chagas, um corolário conceitual imperativo demandaria sempre excluir-se comprometimento orgânico, caracterizando a forma mista da moléstia.

Mais relevante do que a última consideração é reconhecer que a normatização acima seja bastante difícil de se obedecer, no que respeita o aspecto da exploração radiológica digestiva; em moldes clínicos, não há, praticamente, como se justificar o emprego do exame de enema opaco, em paciente chagásico com função intestinal inteiramente normal. Mesmo para finalidades de investigação, é muitas vezes virtualmente impossível convencer o paciente chagásico, disposto a colaborar em projetos envolvendo amplo e extenso protocolo de exploração funcional cardiovascular, a submeter-se ao exame de enema opaco. Talvez, devido a impedimentos dessa ordem, muitos estudos relatados sobre a forma indeterminada da doença de Chagas deixaram de explicitar a realização desse exame, como critério de inclusão dos pacientes; em conseqüência, os resultados dessas investigações não são estrita e confiavelmente aplicáveis ao conhecimento da forma indeterminada da doença de Chagas, conforme definição em uso corrente.

Para ilustrar este conceito, foram revisadas nove publicações consecutivas entre 1975 e 1987, oriundas de um mesmo laboratório, explorando o controle autonômico cardíaco ${ }^{8-16}$. Em nenhuma delas se faz referência explícita a quais os exames radiológicos do tubo digestivo realizados em todos os pacientes. Em quatro desses, todos os pacientes chagásicos estudados em comparação com controles normais são cardiopatas ${ }^{8,10,13}$ mas apenas em três dos trabalhos há menção ao número de pacientes com envolvimento digestivo associado $(10 / 14,11 / 14 \text {, e 8/10 })^{9,10,13}$. Em outros 
dois, menciona-se a ocorrência de um grupo com doença digestiva isolada ${ }^{11}$ e de grupo sem doença clinicamente detectável ("forma indeterminada"), embora se explicite que o estudo radiológico digestivo tenha sido executado "quando indicado" 12 . Ainda em dois outros trabalhos, o foco da investigação foi justamente o controle autonômico cardíaco em pacientes com forma digestiva isolada ${ }^{14,16}$; entretanto, não há explícita comprovação de que, no grupo cardiopata (estudado em comparação), haja sido excluída a doença digestiva associada, pelo exame radiológico colônico, em assintomáticos sob este aspecto. Finalmente, uma última publicação caracteriza bem um grupo de chagásicos com "uma forma indeterminada", sem especificar como teria sido descartada eventual doença digestiva ${ }^{15}$.

Portanto, nesse conjunto de investigações, não se assegura ter sido excluída a concomitância de envolvimento digestivo nos cardiopatas chagásicos. Tampouco a forma indeterminada foi explicitamente caracterizada, com base nos critérios atualmente recomendados. É importante ressaltar que essa constatação não é apanágio do centro de onde provieram as publicações citadas, mas se aplica à vasta maioria das correlatas consultadas.

A indicação rotineira do exame radiológico do cólon para caracterização da forma indeterminada da doença de Chagas é questionável. Além de tratar-se de método diagnóstico muitas vezes mal tolerado pelos pacientes, a prevalência da colopatia isoladamente e sem qualquer sintomatologia associada parece ser baixa ${ }^{17,18}$; existem muitas divergências quanto à interpretação deste exame radiológico em situações limítrofes, em que há pouca ou nenhuma dilatação do cólon ${ }^{19}$. Deve-se mencionar ainda que, enquanto a prevalência da esofagopatia é bem conhecida em áreas endêmicas da doença de Chagas, variando entre 1,2 e 11,2\%, em diferentes estudos ${ }^{20}$, o mesmo não ocorre com a colopatia. Ademais, o envolvimento do cólon parece ocorrer mais tardiamente que o do esôfago na evolução da doença e está freqüentemente associado à esofagopatia ${ }^{17}$. Assim, não parece bem fundamentada a utilização do estudo radiológico do cólon, como método de triagem para identificar eventual envolvimento deste órgão em indivíduos assintomáticos, com estudo radiológico do esôfago normal.

\section{Forma indeterminada versus "normalidade cardiovascular"}

Muitas investigações comprovam a ocorrência de inúmeros distúrbios funcionais em pacientes chagásicos com a forma indeterminada ${ }^{21-30}$. Também estudos com biópsia ou necropsia (em pacientes chagásicos falecidos por morte acidental e, supostamente, cursando na forma indeterminada) relatam anormalidades estruturais cardíacas ${ }^{31-33}$ nessa condição clínica.

Algumas características desses estudos merecem interpretação crítica aprofundada. As alterações estruturais microscópicas, analisadas em material de biópsia, e descritas em $60 \%$ dos pacientes estudados, indigitam a presença de miocardite focal de baixa intensidade. Por técnicas imuno-histoquímicas, há correlação topográfica dos focos inflamatórios com componentes parasitários tissulares. Pelas razões enunciadas, é possível que pelo menos alguns dos pacientes incluídos pudessem ser portadores de anormalidades digestivas, radiologicamente detectáveis, isto é, não se enquadrassem nos critérios estritos, que definem a forma indeterminada. De maneira análoga, não se dispunha sequer do eletrocardiograma em muitos, senão em todos os indivíduos chagásicos necropsiados, após morte acidental, nem havia completa certeza da ausência de sintomas, para classificá-los seguramente como portadores da forma indeterminada.

Os distúrbios funcionais, detectados por meios laboratoriais diversos em pacientes chagásicos indeterminados, comprometem a função diastólica e sistólica ventricular esquerda, a função sistólica ventricular direita, a geração do estímulo sinusal, a condução atrioventricular, o automatismo ventricular e o controle autonômico sinusal. De forma geral, nos estudos assim configurados com as possíveis imprecisões metodológicas citadas, pôde-se verificar que: a) quando diversos exames (e mais particularmente múltiplos testes são realizados no mesmo indivíduo), grupos de pacientes chagásicos na fase indeterminada são praticamente sempre discerníveis de controles não-chagásicos, com base em alguma ou várias anormalidades funcionais e/ou estruturais; b) em contraposição, dentro de qualquer desses relatos, sempre há pacientes chagásicos indeterminados com e sem qualquer anormalidade funcional.

Portanto, em síntese, o envolvimento cardíaco, sendo proteiforme em sua apresentação laboratorial, a ocorrência torna-se individualmente imprevisível, quanto ao distúrbio ou alteração verificados (inclusive com muito variável intensidade). Embora os resultados sugiram, não há rigorosa certeza de sua real e universal ocorrência nos pacientes indeterminados, o que pode decorrer, em parte, da possibilidade de, nos estudos citados, terem sido incluídos muitos pacientes com a forma digestiva da doença.

\section{Forma indeterminada e prognóstico}

Pelas evidências disponíveis, ainda que não-ideais sob o prisma epidemiológico, conclui-se que a forma indeterminada da doença de Chagas se associa a excelente prognóstico $^{3,4,34,35}$, relatando-se mortalidade atribuível à doença, nesses estudos, variando entre 0 e 2,5\% dos casos em seguimento de até 10 anos. Não há, até o presente, evidências de que quaisquer dessas anormalidades cardíacas, funcional e/ou estruturalmente detectadas em pacientes com a forma indeterminada, tenham influência sobre a história natural da moléstia. Ainda mais relevante, não se dispõe de qualquer indício de que essas alterações se associem a mecanismos fisiopatológicos, responsáveis por desencadear a forma cardiopática crônica da doença de Chagas, aspecto esse que parece muito mais dependente das "tempestuosas" relações parasito-hospedeiro e da conseqüente reação imunológica ${ }^{36,37}$.

Há evidência inquestionável de que depressão da 
função sistólica do ventrículo direito possa preceder a instalação de disfunção do esquerdo, em muitos pacientes chagásicos sem cardiopatia aparente (com as formas digestiva ou mesmo indeterminada pelo critério clássico) ${ }^{38,39}$. Esses resultados foram obtidos quando se empregou a ventriculografia nuclear para avaliar a função biventricular de pacientes chagásicos.

Não são claras as razões por que não teriam essas anormalidades da função do ventrículo direito sido descritas em estudos ecocardiográficos bidimensionais de pacientes chagásicos, mas é possível que, para esse propósito, esse método seja particularmente menos propício e sensível. É possível que a câmara ventricular direita exija mais meticulosa e específica atenção quanto ao exame ecocardiográfico bidimensional, para adequada percepção de suas alterações. Paralelamente, deve-se assinalar que o método cineangiográfico de contraste, quando empregado para avaliar pacientes chagásicos, fosse para propósitos clínicos (e.g. exploração da possibilidade de coronariopatia obstrutiva em pacientes com precordialgia) ou mesmo com intuito de investigação, virtualmente, sempre negligenciou a análise da função ventricular direita ${ }^{40}$. Como exceção, constitui a pesquisa recente em chagásicos com taquicardia ventricular e função biventricular explorada cineangiocardiograficamente; embora nesse estudo a origem da taquicardia, raramente se originasse do ventrículo direito, em muitos pacientes a disfunção global ou segmentar desta câmara foi detectada, em associação ou na ausência de comprometimento ventricular esquerdo ${ }^{41}$.

É bastante provável que a disfunção ventricular direita, precocemente instalada, contribua para conferir a peculiar preponderância dos sinais de congestão sistêmica, descrita em pacientes chagásicos com insuficiência cardíaca ${ }^{42,43}$. Entretanto, sua presença, isoladamente, não parece ser incompatível com a manutenção de reserva cardiovascular em níveis de normalidade ${ }^{42}$. Ademais, o impacto prognóstico da disfunção isolada do ventrículo direito não foi ainda determinado.

Resultados recentes ${ }^{44}$ mostraram que pacientes chagásicos com as formas indeterminada, digestiva ou mesmo cardíaca leve (apenas caracterizada pela presença isolada de distúrbio da condução intraventricular do estímulo elétrico) em estudo ecocardiográfico bidimensional, tinham desempenho ventricular esquerdo indiscernível do observado em grupo de voluntários normais. Entretanto, durante estímulo vasopressor com fenilefrina, apresentavam a relação pressão-dimensão ao final da sístole (índice de contratilidade ventricular que independe da pré-carga e incorpora a pós-carga na sua determinação), com valores nitidamente deprimidos, comparativamente ao grupo normal.

Mais significativamente, essa depressão de contratilidade era muito mais acentuada em subgrupo de chagásicos, apresentando alterações mínimas de mobilidade segmentar do ventrículo esquerdo do que naqueles em que o distúrbio de condução elétrica era a única anormalidade a caracterizar o envolvimento miocárdico pela doença. Essas observações são consistentes com envolvimento miocárdico difuso e precoce, expresso apenas em condições de estresse, quando o estado contrátil foi avaliado por metodologia mais sensível; indicam, ainda, que a presença de anormalidades segmentares ao ecocardiograma biplanar constitui fator precoce de depressão contrátil global do ventrículo esquerdo.

Anormalidades contráteis do ventrículo esquerdo, mesmo que mínimas, parecem ter implicação prognóstica não negligível. Resultados preliminares deste laboratório ${ }^{45}$ mostram que pacientes chagásicos com discinergia segmentar do ventrículo esquerdo ao estudo ecocardiográfico bidimensional basal, seguidos por um período médio de 4,6 anos, apresentam progressão mais rápida da disfunção ventricular do que aqueles sem alterações de mobilidade das paredes ventriculares no ecocardiograma basal. Em que pese o caráter ainda preliminar desses resultados e a sua verificação por estudo retrospectivo, torna-se imperativo, no contexto de uma doença inflamatória, como a doença de Chagas, considerar o portador dessas discinergias ventriculares mínimas como cardiopata. Este conceito está em consonância com a noção consolidada em outras afecções cardiovasculares, de que a disfunção ventricular esquerda, regional ou global, constitua marcador prognóstico dos mais relevantes.

Em síntese, o conjunto dos aspectos mencionados oferece base consistente para a presente proposta de revisão do conceito de forma indeterminada da doença de Chagas: 1) que se passe a considerar, além da ausência de sintomatologia e de alterações ao eletrocardiograma e aos estudos radiológicos do tórax e esôfago, a demonstração ecocardiográfica bidimensional de normalidade; 2 ) que seja abolida a exigência de demonstração radiológica por enema opaco de cólon normal em assintomáticos. Seria oportuno especular no momento sobre a eventual possibilidade de esse exame vir a ser substituído por ultra-sonografia abdominal, quando aplicável.

Ressaltamos que o espírito desta proposta encerra o enfoque sobre a função ventricular global e regional, atualmente avaliada pela técnica mais disponível e econômica. É óbvio que se o ecocardiograma não for praticável por motivos técnicos, outros métodos, como a ventriculografia nuclear ou de contraste radiológico, e a ressonância magnética, poderão propiciar a informação diagnóstica aqui sugerida para integrar o conceito de forma indeterminada. Devese enfatizar também a necessidade de, na avaliação ecocardiográfica, focalizar-se específica e criteriosamente, a análise da função ventricular direita.

\section{Implicações sociais e médico-legais, e possível desdobramento terapêutico}

É preciso ter consciência de que, se aplicados os critérios aqui propostos, a prevalência de indivíduos chagásicos, portadores da forma indeterminada, será reduzida em magnitude, ainda a ser estabelecida, mas diretamente relacionada à freqüência com que serão detectadas as anormalidades regionais de contração ventricular.

Em correspondência, mais do que nunca será necessá- 
rio impedir que indivíduos chagásicos com essas formas incipientes de cardiopatia sejam injustificadamente discriminados do ponto de vista social. Embora seu prognóstico possa, aparentemente, com base nas observações preliminares comentadas, ser mais reservado do que o daqueles sem esses distúrbios regionais, esses aspectos carecem de validação ulterior e de esclarecimento quanto ao seu valor a longo prazo. Portanto, para efeitos médico-sociais e de implicações trabalhistas e previdenciárias, esses pacientes deveriam sempre, em base individual, ser protegidos de discriminação indevida, como preconizado para os pertencentes à forma indeterminada, classicamente definida ${ }^{7,19,46-48}$. Em síntese, esses pacientes, ainda que merecedores de seguimento clínico mais meticuloso do que aqueles permanecendo sob a égide de forma indeterminada, necessitarão também ser protegidos de estigmatização social indevida.

É bastante plausível admitir que nesses pacientes, com mínimas alterações da função ventricular regional, seja possível testar o real valor do tratamento etiológico da doença de Chagas. São os que potencialmente mais poderão se beneficiar com tal tipo de tratamento, se a teoria de que a ação inflamatória (diretamente relacionada à persistência parasitária, e mediada e/ou exaltada por agressão auto-imune) mostrar-se correta pela evidência indireta de cunho terapêutico obtida ${ }^{49}$. Urge comprovar cientificamente se os promissores mas ainda muito incipientes resultados dos poucos estudos controlados, apenas parcialmente satisfatórios para testar a hipótese de benefício terapêutico em chagásicos indeterminados ou com mínimas evidências de cardiopatia, deverão ser aplicáveis em larga escala a essa imensa população de pacientes ${ }^{50}$.

Finalmente, é razoável especular que, à semelhança do que se tem demonstrado na disfunção ventricular de outras etiologias, em que a sobrevida e a qualidade de vida a longo prazo são afetadas favoravelmente por inibidores da enzima de conversão da angiotensina e/ou bloqueadores beta-adrenérgicos, quando se intervém precocemente sobre o remodelamento e a discinergia regional incipiente ${ }^{51-56}$, pacientes chagásicos com tais alterações, deixando de constituir o grupo com a forma indeterminada, possam também se beneficiar de medidas terapêuticas similares. Esta hipótese demanda teste científico adequadamente elaborado e executado.

\section{Referências}

1. Chagas C. Estudo sobre a morfologia e o ciclo evolutivo do Schizotrypanum cruzi: agente etiológico de nova entidade mórbida no homem. Mem Inst Oswaldo Cruz 1909; 1: 159-219.

2. Laranja FS. Aspectos clínicos da moléstia de Chagas. Rev Bras Med 1953; 10 : 482-91.

3. Dias JCP. História natural. In: Cançado JR, Chuster M. Cardiopatia Chagásica. Belo Horizonte: Fundação Carlos Chagas, 1985: 99-113.

4. Macedo V. Forma indeterminada da doença de Chagas. JBras Med 1980; 38: 34-40.

5. Décourt LV, Mady C, Sosa FA. Forma indeterminada: conceito e aspectos fisiopatológicos. In: Cançado JR, Chuster M. Cardiopatia Chagásica. Belo Horizonte. Fundação Carlos Chagas 1985: 121-7.

6. Barretto ACP, Mady C. Forma indeterminada da doença de Chagas. Arq Bras Cardiol 1986; 47: 299-302.

7. Oliveira JrW, Salazar LF, Malta J, et al. Análise crítica da forma indeterminada da doença de Chagas. Arq Bras Cardiol 1986; 47: 283-8.

8. Gallo Jr L, Marin-Neto JA, Manço JC, Rassi A, Amorim DS. Abnormal heart rate responses during exercise in patients with Chagas'disease. Cardiology 1975; 60: $147-60$.

9. Marin-Neto JA, Gallo Jr L, Manço JC, Rassi A, Amorim DS. Postural reflexes in chronic Chagas's heart disease. Cardiology 1975; 60: 343-57.

10. Marin-Neto JA, Gallo Jr L, Manço JC, Rassi A, Amorim DS. Mechanisms of tachycardia on standing: studies in normal individuals and in chronic Chagas' heart patients. Cardiovasc Res 1980; 14: 541-50.

11. Amorim DS, Manço JC, Gallo Jr L, Marin-Neto JA. Chagas'heart disease as an experimental model for studies of cardiac autonomic function in man. Mayo Clin Proc 1982; 57(suppl.): 48-60.

12. Junqueira Jr LF, Gallo Jr L, Manço JC, Marin-Neto JA, Amorim DS. Subtle cardiac autonomic impairment in Chagas' disease detected by baroreflex sensitivity testing. Brazilian J Med Biol Res 1985; 18: 171-8.

13. Marin-Neto JA, MacielBC, Gallo Jr L, Junqueira Jr LF, Amorim DS. Effect of parasympathetic impairment on the haemodynamic response to handgrip in Chagas's heart disease. Br Heart J 1986; 55: 204-10.

14. Sousa ACS, Marin-Neto JA, Maciel BC, Gallo Jr L, Barreto-Martins LE, Amorim DS. Use of isometric exercise to demonstrate cardiac parasympathetic impairment in the digestive form of Chagas'disease. Brazilian J Med Biol Res 1987; 20: 781-3.

15. Gallo JrL, Morelo-Filho J, Maciel BC, Marin-Neto JA, Barreto-Martins LE, LimaFilho EC. Functional evaluation of sympathetic and parasympathetic system in Chagas' disease using dynamic exercise. Cardiovasc Res 1987; 21: 922-7.

16. Sousa ACS, Marin-Neto JA, MacielBC, Gallo JrL, Amorim DS. Cardiac parasympathetic impairment in gastrointestinal Chagas' disease. Lancet 1987; I 8539: 985.
17. Rezende JM, Moreira H. Chagasic megaesophagus and megacolon. Historical review and present concepts. Arq Gastroenterol 1988; 25 (special issue): 32-43.

18. Tafuri WL. Patogenia do Megas. In: MDSI. Ed. Tópicos em gastroenterologia 2. Gastroenterologia Tropical: Ed Médica e Científica Ltda., 1991: 163.

19. Dias JCP. The indeterminate form of human chronic Chaga's disease: a clinical epidemiological review. Rev Soc Bras Med Trop 1989; 22: 147-56.

20. Castro C, Rezende JM, Camargo M, Prata A, Macedo V. Prevalência de esofagopatia chagásica no município de Mambaí, Goiás-Brasil. Rev Soc Med Trop 1987; 20: 13-7.

21. Macedo V, Martinelli G, Alves PS, et al. Cicloergometria na forma indeterminada da doença de Chagas. Arq Bras Cardiol 1979; 32(supl. 1): 47.

22. Martins N, Fernandes L, Seixas T, etal. Teste cicloergométrico na forma indeterminada ou digestiva da doença de Chagas. Arq Bras Cardiol 1983;41(supl. 1): 173.

23. Almeida JWR, Yasuda MAS, Amato Neto V, et al. Estudo da forma indeterminada da doença de Chagas através da eletrocardiografia dinâmica. Rev Inst Med Trop 1982; 24: 222-8.

24. Marins N, Flores AP, Seixas TN, et al. Eletrocardiografia dinâmica em Chagas na forma indeterminada ou sem cardiopatia aparente. Arq Bras Cardiol 1983; 39: 303-7.

25. Mady C, Arteaga-Fernandez E, Moffa P, et al. Forma indeterminada da doença de Chagas: análise comparativa entre o eletrocardiograma e vetocardiograma. Arq Bras Cardiol 1982; 31(supl. 1): 41.

26. Ortiz J. Aspectos ecocardiográficos. In: Cançado JR, Chuster M. Cardiopatia chagásica. Belo Horizonte: Fundação Carlos Chagas, 1985: 165-8.

27. Garzon SAC, Lorga AM, Greco OP, et al. O cardiograma apexiano na lesão apical da cardiopatia chagásica crônica. Arq Bras Cardiol 1973; 1: 75.

28. Mady C, Moraes AV, Galinno N, et al. Estudo hemodinâmico na forma indeterminada da doença de Chagas. Arq Bras Cardiol 1982; 38: 271.

29. Marins N, Silva CP, Motta VP, et al. Estudo hemodinâmico em indivíduos chagásicos sem cardiopatia aparente. Arq Bras Cardiol 1981; 37: 463.

30. Décourt LV, Sosa EA, Pileggi F. Estudos eletrofisiológicos cardíacos na forma indeterminada da doença de Chagas. Arq Bras Cardiol 1981; 36: 227-34.

31. Pereira-Barretto AC, Mady C, Arteaga-Fernandez E, et al. Right ventricular endomyocardial biopsy in chronic Chagas' disease. Am Heart J 1986; 10: 665-70.

32. Lopes ER, Chapadeiro E, Andrade ZA, et al. Anatomia patológica de corações de chagásicos assintomáticos falecidos de modo violento. Mem Inst Oswaldo Cruz 1981; 76: 189-97.

33. Prata A, Lopes ER, Chapadeiro E. Características da morte súbita tida como nãoesperada na doença de Chagas. Rev Soc Bras Med Trop 1986; 19: 9-12.

34. Dias JCP, Kloetzel K. The prognostic value of the electrocardiographic features of chronic Chagas' disease. Rev Inst Med Trop São Paulo 1968; 10: 158-62. 
35. Coura JR, Abreu LL, Pereira JB, Willcox HP. Morbidade da doença de chagas.IV. Estudo longitudinal de dez anos em Pains e Iguatama, Minas Gerais. Mem Inst Oswaldo Cruz 1985; 80: 73-80.

36. Higuchi ML. Chronic chagasic cardiopathy: the product of a turbulent host-parasite relationship. Rev Inst Med Trop São Paulo 1997; 39: 53-60.

37. Cunha-Neto E, Coelho VPC, Guilherme L, Fiorelli A, Stolf N, Kalil J. Autoimmunity in Chagas's disease: Identification of cardiac myosin-B13 Trypanosoma cruzi protein crossreactive T cell clones in heart lesions of a chronic Chagas' cardiomyopathy patient. J Clin Invest 1996; 98: 1709-12.

38. Marin-Neto JA, Marzullo P, Sousa ACS, et al. Radionuclide angiographic evidence for early predominant right ventricular involvement in patients with Chagas' disease. Can J Cardiol 1988; 4: 231-6.

39. Marin-Neto JA, Bromberg-Marin G, Pazin Filho A, Simões MV, MacielBC.Cardiac autonomic impairment and early myocardial damage involving the right ventricle are independent phenomena in Chagas' disease. Int J Cardiol 1998; 65: 261-9.

40. Maciel BC, Almeida-Filho OC, Schmidt A, Marin-Neto JA. Ventricular function in Chagas' heart disease. São Paulo Medical Journal/RPM 1995; 13: 814-20.

41. Sarabanda AVL, Marin-Neto JA, Simões MV, Figueiredo G, Pintya A, Sosa EA. Electrophysiologic, angiographic and myocardial perfusion characteristics that differentiate between sustained and nonsustained ventricular tachycardia in Chagas'heart disease. PACE 2001; 24: 723.

42. Marin-Neto JA, AndradeZA. Por que é usualmente predominante a insuficiência cardíaca direita na moléstia de Chagas? Arq Bras Cardiol 1991; 57: 181-3.

43. Marin-Neto JA, Simões MV, Maciel BC. Aspectos peculiares da fisiopatologia da insuficiência cardíaca na doença de Chagas. Rev Soc Cardiol Est São Paulo 1998; 8: 243-52.

44. Almeida-Filho OC, Schmidt A, Marin Neto JA, Maciel BC. Minor segmentar dyssynergy reflects extensive myocardial damage and global left ventricle dysfunction in chronic Chagas's disease. J Am Soc Echocardiogr, 2001 (in press).

45. Pazin-Filho A, Almeida-Filho OC, Furuta MS, et al. Prognostic implications of minor segmental wall motion abnormalities in patients with Chagas' disease. $\mathrm{J}$ Am Coll Cardiol 1998; 31(5-suppl. C): 339C.
46. Prata A. Implicações epidemiológicas e sócio-econômicas da doença de Chagas. Brasília Médica 1973; 9: 69-71.

47. Porto CC, Rassi A, Faria GHDC. Aspectos sócio-econômicos e médico-trabalhistas. In: Cançado JR, Chuster M. Cardiopatia Chagásica. Belo Horizonte: Fundação Carlos Chagas, 1985: 362-6.

48. Oliveira Jr W. Forma indeterminada da doença de Chagas: implicações médicotrabalhistas. Arq Bras Cardiol 1990; 54: 89-91.

49. Rassi Júnior A, Marin-Neto JA. Estado da Arte. Cardiopatia chagásica crônica. Rev Soc Cardiol Est São Paulo 2000; 10: vi-xxxii.

50. Villar JC, Marin-Neto JA, Ebrahim S, YusufS. Trypanocidal therapy for chronic asymptomatic Trypanosoma cruzi infection: a systematic review. Cocrrane Database Syst Rev 2002(1): CD 003463.

51. The SOLVD Investigators. Effect of enalapril on survival in patients with reduced left ventricular ejection fraction and congestive heart failure. N Engl J Med 1991; 325: 293-302.

52. ISIS-4 Collaborative Group. ISIS-4: a randomized factorial trial assessing early oral captopril, oral mononitrate, and intravenous magnesium sulphate in 58,050 patients with suspected acute myocardial infarction. Lancet 1995; 345: 669-85.

53. Packer M, Colluci WL, Bristow MR, et al. US carvedilol Heart Failure Study group. The effect of carvedilol on morbidity and mortality in patients with chronic heart failure. N Engl J Med 1996; 334: 1349-55.

54. FlatherMD, Yusuf S, Kober L, et al on behalf of the ACE-Inhibitor Myocardial Infarction Collaborative Group. Systematic overview of individual patient data from the large randomized trials of long term ACE-inhibitor therapy in patients with heart failure or left ventricular dysfunction. Lancet 2000; 355: 1575-81.

55. Remme J, on behalf of the Steering Committee and Investigators. The Carvedilol and ACE-Inhibitor Remodeling Mild Heart Failure Evaluation Trial (CARMEN)-rationale and design. Cardiovasc Drugs Ther 2001; 15: 69-71.

56. The CAPRICORN Investigators. Effect of carvedilol on outcome after myocardial infarction patients with left-ventricular dysfunction: the CAPRICORN randomised trial. Lancet 2001; 357: 1385-90.

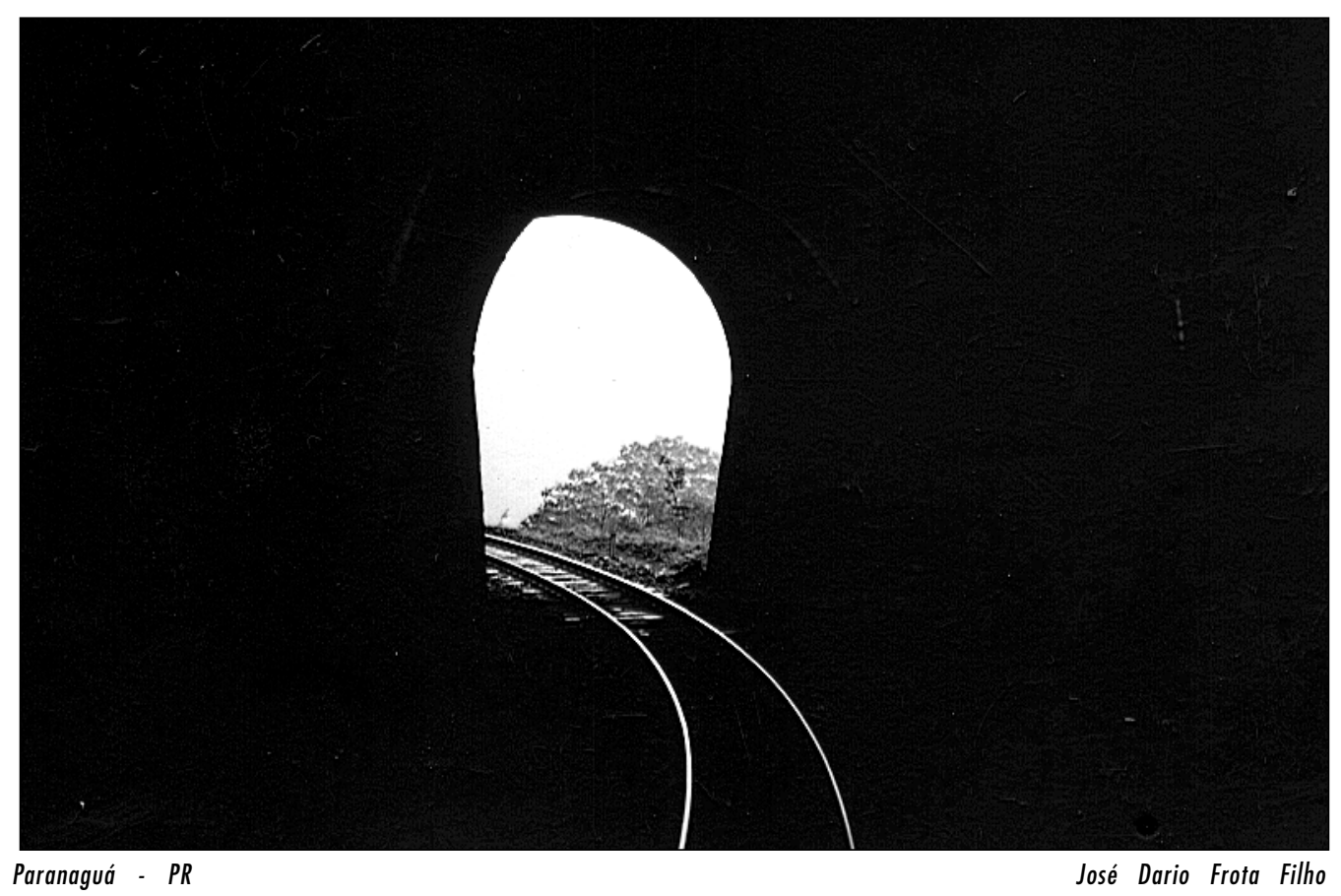

Editor da Seção de Fotografias Artísticas: Cícero Piva de Albuquerque

Correspondência: InCor - Av. Dr. Enéas C. Aguiar, 44 - 05403-000 - São Paulo, SP - E-mail: delcicero@incor. usp.br 